\title{
Chip-Scale Frequency Comb Generators for High-Speed Communications and Optical Metrology
}

\author{
C. Koos ${ }^{\mathrm{a}, \mathrm{b}, *}$, T. J. Kippenberg ${ }^{\mathrm{c}}$, L. P. Barry ${ }^{\mathrm{d}}$, A. Ramdane ${ }^{\mathrm{e}}$, F. Lelarge ${ }^{\mathrm{f}}$, W. Freude ${ }^{\mathrm{a}, \mathrm{b}}$, \\ P. Marin ${ }^{\mathrm{a}}$, J. N. Kemal ${ }^{\mathrm{a}}$, C. Weimann ${ }^{\mathrm{a}}$, S. Wolf ${ }^{\mathrm{a}}$, P. Trocha ${ }^{\mathrm{a}}$, J. Pfeifle ${ }^{\mathrm{a}}$, M. Karpov' ${ }^{\mathrm{c}}$,

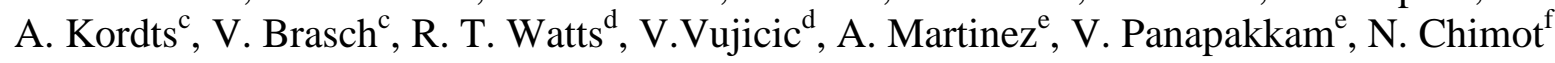 \\ ${ }^{a}$ Institute of Photonics and Quantum Electronics (IPQ), Karlsruhe Inst. of Technology (KIT), 76131 Karlsruhe, Germany \\ ${ }^{\mathrm{b}}$ Inst. of Microstructure Technology (IMT), Karlsruhe Inst. of Techn. (KIT), 76344 Eggenstein-Leopoldshafen, Germany \\ ${ }^{\mathrm{c}}$ Ecole Polytechnique Fédérale de Lausanne (EPFL), 1015 Lausanne, Switzerland \\ ${ }^{\mathrm{d}}$ The Rince Institute, School of Electronic Engineering, Dublin City University, Glasnevin, Dublin 9, Ireland \\ ${ }^{\mathrm{e}}$ Laboratoire de Photonique et Nanostructures, CNRS UPR20, Marcoussis, France \\ ${ }^{\mathrm{f}}$ III-V Labs, Marcoussis, France \\ *christian.koos@kit.edu
}

\begin{abstract}
Chip-scale frequency comb sources are key elements for a variety of applications, comprising massively parallel optical communications and high-precision optical metrology. In this talk, we give an overview on our recent progress in the area of integrated optical comb generators and of the associated applications. Our experiments cover modulator-based comb sources, injection locking of gain-switched laser diodes, quantum-dash mode-locked lasers, as well as Kerr comb sources based on cavity solitons. We evaluate and compare the performance of these devices as optical sources for massively parallel wavelength division multiplexing at multi-terabit/s data rates, and we report on comb-based approaches for high-precision distance metrology.
\end{abstract}

Keywords: Frequency combs, optical communications, optical interconnects, optical metrology, integrated photonics, Kerr frequency combs, cavity solitons

\section{SUMMARY}

Frequency combs are key components for a wide variety of applications, ranging from high-precision metrology to highspeed optical communications. However, to unlock the full potential of such concepts, comb sources must be combined with miniaturized photonic circuits that can be efficiently realized on industrially mature integration platforms such as silicon photonics [1], [2], indium phosphide [3], or silicon nitride [4], [5]. This is impeded by the fact that the vast majority of currently available comb sources are still bulky, expensive and mechanically sensitive so that they cannot be integrated into compact and robust chip-scale systems. In this talk, we give an overview on our recent progress in highspeed communications and optical metrology using chip-scale comb generators that can be efficiently combined with photonic integrated circuits either in a monolithic or in a hybrid approach.

High-speed optical links rely on coherent transmission using higher-order modulation formats along with advanced wavelength-division multiplexing (WDM) schemes. In this context, frequency combs are perfectly suited as multiwavelength light sources at the transmitter and as multi-wavelength local oscillators (LO) at the receiver. Unlike carriers derived from a bank of individual laser modules, the tones of a comb are intrinsically equidistant in frequency, thereby enabling transmission of optical data signals at highest spectral efficiency [6]. In addition, stochastic frequency variations of the carriers are strongly correlated, which enables efficient compensation of impairments caused by nonlinearities of the optical fiber in long-distance transmission [7]. To demonstrate the viability of optical frequency combs for high-speed coherent communications, we have performed a set of experiments that exploit different comb generator concepts at the transmitter as well as at the receiver.

In a first set of experiments, we demonstrate frequency comb generation using silicon-organic hybrid (SOH) electrooptic modulators, thereby achieving line rates up to $1.152 \mathrm{Tbit} / \mathrm{s}$ transmitted on 9 optical carriers [8]. SOH integration 
combines silicon photonic slot waveguides with organic electro-optic cladding materials to realize highly efficient modulators [9], [10]. These devices enable large modulation depths at moderate drive voltages, thereby generating frequency combs from a single continuous-wave (cw) laser line.

In a second set of experiments, we use injection locking of gain-switched laser diodes to generate frequency combs [11]. When used as a multi-wavelength optical source at the transmitter, these so-called gain-switched combs sources (GSCS) enable line rates of more than 2 Tbit/s on 24 optical carriers. We also demonstrate that GSCS can not only act as a transmitter light source, but also as multi-wavelength local oscillator, thereby exploiting the scalability advantages of optical frequency combs also at the receiver side [12]

In a third set of experiments, we use quantum-dash mode-locked laser diodes (MLLD) as frequency comb sources. These devices exhibit rather large optical linewidths, which either requires dedicated phase-noise reduction schemes [13], selfhomodyne detection [14], or digital phase tracking [15] to enable coherent communications with higher-order modulation formats at low symbol rates. However, at high symbol rates of, e.g., $40 \mathrm{GBd}$ or more, carrier phase noise is less detrimental, and no additional measures are needed. In a WDM experiment with 52 channels, we demonstrate transmission of an aggregate line rate of $8.32 \mathrm{Tbit} / \mathrm{s}$ (net data rate of $7.74 \mathrm{Tbit} / \mathrm{s}$ ) over a transmission distance of $75 \mathrm{~km}$ [15]. More recently, we have shown that at sufficiently high symbol rates, MLLD can also be used as multiwavelength LO, despite their comparatively large optical linewidth [16].

Finally, a fourth set of experiments is dedicated to comb sources that exploit Kerr nonlinearities in integrated siliconnitride microcavities fabricated by a so-called photonic Damascene process for stress control [17]. In a first experiment, we demonstrated coherent communications using a Kerr frequency comb source, thereby achieving line rates of up to $1.44 \mathrm{Tbit} / \mathrm{s}$ on 20 carriers [18]. More recently, we succeeded in increasing the transmission speed to data rates beyond $50 \mathrm{Tbit} / \mathrm{s}$ by using highly stable dissipative cavity soliton Kerr combs that provide hundreds of spectral carriers at infrared telecommunication wavelengths [19] - [23]. This corresponds to the highest data rate achieved to date using a chip-scale frequency comb source. In our experiments, we use two interleaved Kerr combs to transmit data on a total of 179 individual optical carriers that span the entire telecommunication C and L bands. Equally important, we demonstrate coherent detection of a WDM data stream by using a Kerr soliton comb as a multi-wavelength $\mathrm{LO}$ at the receiver. These results prove the tremendous technological potential of chip-scale microresonator soliton comb sources in high-speed communications. In combination with advanced spatial multiplexing schemes [24] and highly integrated silicon photonic circuits [2], microresonator soliton combs may bring chip-scale petabit/s transceiver systems into reach.

Besides optical communications, optical metrology can also benefit greatly from combining photonic integrated circuits with chip-scale frequency comb generators. This applies, e.g., to high-precision distance metrology [25], where frequency combs allow to maintain high sampling speeds and high precision even when measuring the distance to scattering technical surfaces [26]. The receiver system of such metrology schemes can be efficiently integrated on silicon-on-insulator chips [27]. To realize fully integrated optical metrology systems, these devices can be connected to chip-scale comb-generators comb by photonic wire bonds [28], [29]. While high-speed optical communications is currently the most important driver for co-integration of photonic circuits and chip-scale comb generators, we believe that optical metrology and sensing will also benefit greatly from the tremendous technological advances that the field has experienced in recent years.

\section{Acknowledgements}

This work was supported by the European Research Council (ERC Starting Grant 'EnTeraPIC', number 280145), by the Alfried Krupp von Bohlen und Halbach Foundation, by the EU-FP7 projects BigPipes and Phoxtrot, by the Helmholtz International Research School for Teratronics (HIRST), by the Karlsruhe School of Optics \& Photonics (KSOP), by the Erasmus-Mundus Joint Doctorate Program EUROPHOTONICS, and by the Karlsruhe Nano-Micro Facility (KNMF). We acknowledge financial support by the Deutsche Forschungsgemeinschaft (DFG) through the Collaborative Research Center "Wave Phenomena: Analysis and Numerics" (CRC 1173). Samples for Kerr comb sources were grown and fabricated in the EPFL CMI-center for nanofabrication.

\section{References}

[1] Thomson, D.; Zilkie, A.; Bowers, J. E.; Komljenovic, T.; Reed, G. T.; Vivien, L.; Marris-Morini, D.; Cassan, E.; Virot, L.; Fédéli, J.-M.; Hartmann, J.-M.; Schmid, J. H.; Xu, D.-X.; Boeuf, F.; O’Brien, P.; Mashanovich, G. Z.; Nedeljkovic, M.: "Roadmap on silicon photonics", Journal of Optics 18, 073003 (2016)

[2] Dai, D.; Bowers, J. E.: "Silicon-based on-chip multiplexing technologies and devices for Peta-bit optical interconnects”. Nanophotonics 3, 283-311 (2014). 
[3] Binetti, P. R. A.; Lu, M.; Norberg, E. J.; Guzzon, R. S.; Parker, J. S.; Sivananthan, A.; Bhardwaj, A.; Johansson, L. A.; Rodwell, M. J.; Coldren, L. A.: "Indium Phosphide Photonic Integrated Circuits for Coherent Optical Links," IEEE Journal of Quantum Electronics 48, 279 - 291 (2012).

[4] Rahim, A. et al.: "Expanding the Silicon Photonics Portfolio with Silicon Nitride Photonic Integrated Circuits," Journal of Lightwave Technology DOI: 10.1109/JLT.2016.2617624 1 (2016)

[5] Zhu, S.; Lo, G.Q.; Kwong, D.L.: "Silicon nitride based plasmonic components for CMOS back-end-of-line integration," Opt. Express 21, 23376-23390 (2013)

[6] Hillerkuss, D. et al. "Single-Laser 32.5 Tbit/s Nyquist WDM Transmission,” J. Opt. Commun. Netw. 4, 715-723 (2012).

[7] Temprana, E. et al. “Overcoming Kerr-induced capacity limit in optical fiber transmission,” Science 348, 14451448 (2015).

[8] Weimann, C. et al. "Silicon-organic hybrid (SOH) frequency comb sources for terabit/s data transmission," Opt. Express 22, 3629-3637 (2014)

[9] Koos, C. et al.: "Silicon-Organic Hybrid (SOH) and Plasmonic-Organic Hybrid (POH) Integration,” J. Lightw. Technol. 34, 256-268 (2016)

[10] Koeber, S et al.: “Femtojoule electro-optic modulation using a silicon-organic hybrid device,” Light: Science \& Applications 4, e255 (2015)

[11] Pfeifle, J. et al.: “Flexible terabit/s Nyquist-WDM super-channels using a gain-switched comb source,” Opt. Express 23, 724-738 (2015)

[12] Kemal, J. N.; Pfeifle, J.; Marin-Palomo, P.; Gutierrez Pascual, M. D.; Wolf, S.; Smyth, F.; Freude, W.; Koos, C.: "Multi-wavelength coherent transmission using an optical frequency comb as a local oscillator,” Opt. Express 24, 25432--25445 (2016)

[13] Pfeifle, J. et al. "Simultaneous phase noise reduction of 30 comb lines from a quantum-dash mode-locked laser diode enabling coherent Tbit/s data transmission,” Optical Fiber Communication Conference (OFC'15), Los Angeles (CA), USA, 22 - 26 March , Paper Tu3I.5. (2015)

[14] Pfeifle, J. et al. "Coherent terabit communications using a quantum-dash mode-locked laser and self-homodyne detection,” Optical Fiber Communication Conference (OFC'15), Los Angeles (CA), USA, 22 - 26 March, Paper W2A.19. (2015)

[15] Marin, P.; Pfeifle, J.; Kemal, J. N.; Wolf, S.; Vijayan, K.; Chimot, N.; Martinez, A.; Ramdane, A.; Lelarge, F.; Freude, W.; Koos, C.: "8.32 Tbit/s coherent transmission using a quantum-dash mode-locked laser diode,” Conf. on Lasers and Electro-Optics (CLEO'16), San Jose (CA), USA, June 5-10, 2016. Paper STh1F.1

[16] Kemal, J. N.; Marin, P.; Trocha, P.; Wolf, S.; Panapakkam, V.; Ramdane, A.; Randel, S.; Freude, W.; Koos, C.: "WDM Transmission Using Quantum-Dash Mode-Locked Laser Diodes as Multi-Wavelength Source and Local Oscillator,” Optical Fiber Communication Conference (OFC 2017), Los Angeles (CA), USA, March 19-23, 2017.

[17] Pfeiffer, M. H. P.; Kordts, A.; Brasch, V.; Zervas, M.; Geiselmann, M.; Jost, J. D.; Kippenberg, T.; J.: "Photonic Damascene process for integrated high-Q microresonator based nonlinear photonics," Optica 3, 20-25 (2016)

[18] Pfeifle, J. et al. "Coherent terabit communications with microresonator Kerr frequency combs," Nature Photon. 8, 375-380 (2014)

[19] Pfeifle, J. et al.: "Full C and L-band transmission at 20 Tbit/s using cavity-soliton Kerr frequency combs,” Conf. on Lasers and Electro-Optics (CLEO'15), San Jose (CA), USA, May 10-15, 2015. Paper STh5C.8

[20] Marin, P. et al.: "50 Tbit/s massively parallel WDM transmission in C and L band using interleaved cavity-soliton Kerr combs,” Conf. on Lasers and Electro-Optics (CLEO 2016), San Jose (CA), USA, June 5-10, 2016. Paper STu1G.1

[21] Brasch et al., "Photonic chip-based optical frequency comb using soliton-induced Cherenkov radiation,” Science 22, 357-360 (2016)

[22] Marin, P. et al.: "34.6 Tbit/s WDM transmission using soliton Kerr frequency combs as optical source and local oscillator,” 42nd European Conf. Opt. Commun. (ECOC 2016), Düsseldorf, Germany, Sept. 18 -22, 2016, Paper Tu.3.E.3

[23] Marin, P. et al.: “Microresonator solitons for massively parallel coherent optical communications,” arXiv.org; arXiv:1610.01484v3 [nlin.PS] (2016)

[24] Bozinovic, N et al.: "Terabit-scale orbital angular momentum mode division multiplexing in fibers,” Science $\mathbf{3 4 0 ,}$ 1545-1548 (2013).

[25] Weimann, C et al.: "Measurement of Length and Position with Frequency Combs," 23rd Congress of the International Commission for Optics (ICO 23), April 2015, published in J. Phys.: Conf. Ser. 605, 012030 (2015) 
This article has been accepted for publication in Proc. SPIE Nonlinear Frequency Generation and Conversion: Materials and Devices XVI, (LASE-SPIE'17), San Francisco (CA), USA, Jan. 28 - Feb. 2, paper 10090-15 (2017) (invited)

DOI: http://dx.doi.org/10.1117/12.2256465

[26] Weimann, C. et al.: "Fast high-precision distance measurements on scattering technical surfaces using frequency combs,” Conf. on Lasers and Electro-Optics (CLEO'13), San Jose (CA), USA, June 9-14, 2013. Paper CTu2I.3

[27] Weimann, C. et al.:"Silicon photonic integrated circuit for fast distance measurement with frequency combs," Conf. on Lasers and Electro-Optics (CLEO'14), San Jose (CA), USA, June 8-13, 2014. Paper STh4P.3

[28] Lindenmann, N. et al.: “!Connecting silicon photonic circuits to multicore fibers by photonic wire bonding," Journal of Lightwave Technology 33, 755 - 760 (2015)

[29] Lindenmann, N. et al.:’Photonic wire bonding: a novel concept for chip-scale interconnects,” Opt. Express 20, 17667-17677, (2012) 